\title{
Care and education in adolescents with sleep and eating disorders: Experience of the Mediterranean Adolescent Center
}

\author{
F. Bat-Pitault ${ }^{1,2}$, S. Campredon', D. Da Fonseca ${ }^{1,2}$ \\ 1 AP-HM, Child Psychiatry Department, Salvator Hospital, 13009 Marseille, France \\ 2 Aix-Marseille University, Timone Institute of Neuroscience, CNRS, 13005 Marseille, France
}

\begin{abstract}
Eating disorders and school refusal behavior with delayed sleep phase are pathologies associated with very different or even inverse school problems. It is through these two prisms that we will develop the idea of school care, which is being set up in the innovative framework of the Mediterranean Area of the Adolescent, integrating a National Education Unit within the Child and Adolescent Psychiatry unit.

This specific care seems to us interesting in the adolescent because it allows a work in "natural» environment of certain difficulties related to the different psychiatric disorders and seems to us as a help to the revival of the adolescent process.
\end{abstract}

\section{KEYWORDS}

Eating disorders, school refusal behavior, delayed sleep phase, school

\section{INTRODUCTION}

Adolescence is a period full of physical and psychological changes, and it is a phase during which several psychosomatic disorders are prone develop ${ }^{21}$.

At this life stage, schooling can help individuals to develop a number of important processes, such as the need for separation-individuation, the empowerment of parents, social relations within a peer group as well as with peers of the opposite sex, and the acquisition of quality hypotheticaldeductive reasoning.

Therefore, when certain disorders disrupt the smooth progress of adolescence, the young individual's relationships with the school are modified, and their satisfactory reintroduction into education therefore becomes both a care objective and a healing aid.

This relationship between care and schooling is developed daily in the Espace

\section{Address for correspondence:}

This is an Open Access article distributed under the terms of the Creative Commons Attribution License (http://creativecommons.org/licenses/by/4.0), which permits unrestricted use, distribution, and reproduction in any medium, provided the original work is properly cited. 
Méditerranéen de I'Adolescent (EMA) [Mediterranean Center for Adolescents] at the University Hospital of Marseille with the idea that school is essential for the proper development of adolescents, and that schooling can provide a genuine care structure in the context of overall care for some young people. We will see how this modality of care, which was very important

\section{EATING DISORDERS AND SCHOOLING}

\section{Management of eating disorders in adolescence}

The primary adolescent eating disorders are anorexia and bulimia, which show prevalences of $0.3 \%$ and $0.9 \%$, respectively, and $1 \%$ and $2 \%$, respectively, among males and females ${ }^{12,19}$. These disorders affect girls more than boys with a sex ratio of 10:1 in adulthood, 19:1 in older adolescents, and 2.33:1 in prepubescent adolescents ${ }^{25}$. However, male anorexia is becoming more common, with a prevalence of $0.03 \% 14$ in adolescents, although it is probably still being underestimated ${ }^{8}$. Two peaks of onset for anorexia, at 10 12 years and 15-18 years, are known, which makes adolescents a high-risk population, especially if they practice any competitive sports or have a somatic pathology that complicates their diet, such as type-1 diabetes. Screening must therefore be conducted early and targeted on this population to improve the prognosis of patients ${ }^{18}$ because the somatic complications can be significant. The mortality rate is among the highest of psychiatric pathologies to Professor Rufo, and to his successor today, Professor Da Fonseca, is being implemented at the EMA in an innovative unit integrating a National Education Unit within the adolescent psychiatry department. Here, we will focus on how this care work in connection with schooling differs for our adolescents with eating disorders and sleep disorders.

$(5 \%-15 \% \text { for anorexia })^{23}$ and the risk of chronic disease is high"1. The management protocol recommended by the HAS [French High Authority for Health] in 2010 must be multidisciplinary, conducted early, well-coordinated, and may require full-time hospitalization according to predefined criteria ${ }^{18}$.

\section{Relationship to education of patients with eating disorders}

A large proportion of adolescents with eating disorders do not have any school-related problems and even do well in school ${ }^{30}$. In many patients, excessive perfectionism, a need for control, and a pathological focus on school work result in improved outcomes during illness, which contributes to the somewhat caricatured stereotype of these patients as "first in class." Thus, improved school performance for an adolescent who is losing weight, who is becoming more isolated, and has changed their diet should therefore be a cause for alarm. It is important to keep in mind that this typical "good student" profile is not the rule in these 
pathologies and that these pathologies mainly induce a certain amount of cognitive changes and impairments, the outcome of which after the cure is still uncertain and are accompanied by a certain amount of comorbidities in terms of performance anxiety and altered self-esteem, which can also be obstacles to the academic success of these patients. International studies show that many patients with anorexia have a cognitive profile marked by a lack of cognitive flexibility and central coherence ${ }^{32}$; in addition, malnutrition modifies the content of thought by focusing it on the diet and alters the reflexive capacities, with severe asthenia, diminished attention span, and psychomotor slowdown ${ }^{18}$. In terms of comorbidities, self-esteem disorders are relatively common in patients with an eating disorder ${ }^{1}$, and these lead to performance anxiety, thereby severely affecting academic performance. Considering these difficulties in relation to the eating disorders, we can understand better why these patients experience academic difficulties even if it is compensated for in some patients and does not necessarily result in declining grades. As a result of these difficulties, many adolescents have a deterioration in their learning capacities and lower final grades. If the studies show an overall impairment in the quality of life of patients with eating disorders ${ }^{10}$, the disability generated seems particularly significant for students ${ }^{33}$. Finally, Byrne et al. ${ }^{3}$ estimate that just under a third of high school students with eating disorders report a significant clinical disability including academic difficulty. Some factors, such as the association of excessive perfectionism with emotional regulation disorders could, according to these authors, be predictive of this educational and professional disability ${ }^{3}$.

\section{Specificity of the joint care-school management of these disorders within the EMA}

Given the significance given by these patients to education, we have made the decision at the EMA to integrate care as quickly as possible, depending, of course, on the patient's somatic state, into the school to create a "natural" work environment for adolescents with cognitive disorders. This will be coordinated with the cognitive treatment proposed by our neuropsychologist as well as therapy for perfectionism, self-esteem disorder, and performance anxiety proposed by our clinical psychologist. This school care is enabled by multidisciplinary meetings bringing together caregivers, school nurses, at the interface of care and schooling and to improve care by extending it in a classic classroom environment in a location separate from the full-time hospitalization service, while being more reassuring because it is small in size and located within the hospital.

Finally, this work is completed through a measured, gradual recovery program accompanied by schooling in their home institution as part of a sequential hospitalization of adolescents. This allows a link between schooling and National Education Unit staff both within the hospital and outside, in the school of origin, to allow for the discharge from the health care institution to the most favorable school possible, so as to decrease the school stress and potential relapse factors. 


\section{SLEEP DISORDERS AND SCHOOLING}

\section{Sleep disorders in adolescence}

In our modern societies, the prevalence of sleep disorders is high with one-third of the general population complaining of poor sleep ${ }^{27}$. Adolescents are not spared as this complaint, and it affects $13 \%$ boys and $17 \%$ girls. The major consequence of this poor sleep is a chronic sleep deficit that leads to between $20 \%$ and $25 \%$ of the population having a total sleep time (TST) of $<6 \mathrm{~h}$ on weekdays ${ }^{29}$. One of the main sleep disorders responsible for this sleep deficit at this stage of life is a circadian rhythm disorder called delayed sleep phase syndrome, which corresponds to a pathological accentuation of the physiological decline in the amount of sleep in adolescence ${ }^{6}$ because of, among other things, using multimedia screens just before bedtime ${ }^{4.26}$ and doing homework too late in the evening. This delayed sleep phase syndrome, which remains undetected in 7\%-16\% adolescents ${ }^{7,15}$, coupled with the need for an early rise imposed by school rhythms ${ }^{17}$, results in decreased sleep duration and chronic sleep deficit in adolescents. In addition to this circadian rhythm disorder, sleep deficit may also be secondary to sleeplessness, which is present in $20 \%-$ $26 \%$ adolescents, more frequently in girls ${ }^{15}$ because of anxiety, depression, or substance abuse disorders ${ }^{16}$.

\section{Relationship to the patient's education with sleeping disorders}

\section{School consequences of sleep disorders}

Sleep disorders, through the sleep deficit they induce, have negative consequences on the adolescent's schooling, particularly on their academic performance ${ }^{34}$. Therefore, excessive daytime sleepiness, described as mainly secondary to the chronic reduction of sleep time, is present in 30\% French adolescents, with direct consequences in terms of schooling leading to a decrease in performance and an increase in school absenteeism ${ }^{24}$. At the cognitive level, even if the literature does not offer complete picture of the cerebral maturation in adolescence ${ }^{5}$, sleep disturbances because decreased sleep time are clearly harmful and alter learning, attention, and memory processes. ${ }^{22}$ and neural plasticity fundamental to the successful completion of their education. Finally, sleep disturbances can alter schooling by increasing anxiety-depressive symptoms, lowering the positive emotions felt ${ }^{31}$, mimicking a clinical picture of attention deficit disorder with or without hyperactivity ${ }^{9}$ or by contributing to the onset of school refusal behavior (SRB) because of an initial morning awakening difficulty.

\section{Delayed sleep phase syndrome associated with SRB}

Beyond the academic consequences of the TST reduction induced by sleep disorders, which are reversible by sleep management to decrease sleep defi$\mathrm{Cit}^{2}$, a common problem in sleep-related counseling that many practitioners encounter is the inability to implement effective sleep management in adolescents who are consulting for a delayed sleep phase syndrome, which "prevents them from going to school or leads to at least some school absences. The difficulty then lies in the 
emphasis, often unconsciously, of a somatic problem by a teenager who in fact has a psychological difficulty that prevents them from attending school regularly, which is then called SRB as defined by Berg in 1997. It seems important to specify here that this SRB is very often masked by different types of somatization ${ }^{20}$, often multidisciplinary, which delays its diagnosis and its management and can be especially effective if noticed early ${ }^{13}$. It is then a management of the disorders underlying the diagnosis of SRB-including social anxiety, separation anxiety ${ }^{28}$, depressive elements, learning disabilities, or other psychiatric pathologies. This will allow, in close collaboration with the school and the family, the resumption of progressive schooling and the management the delayed sleep phase syndrome, which can then be improved with classical somnological treatment.

\section{Specificity of joint care-education management of delayed sleep phase syndrome associated with SRB within the EMA}

The management of SRB within the EMA is probably the best way to characterize the work of this National Education structure installed within a care facility. The National Education Unit works closely with the day hospital to ensure that our child psychiatry department is equipped to admit and treat patients who are still too symptomatic to be managed by a care-study protocol, especially considering that such children need to be treated with personalized care and schooling in such complex psychological situations.
Conventionally, the recommendations propose ambulatory and close management of SRB, sometimes using a home-based hospitalization system. In many situations it is necessary to institute full-time institutional care or day hospital care to provide individual and family psychotherapy, as well as group care in therapeutic workshops, and then help with school resumption: Academic recovery should occur in parallel with support in the school. But when care is needed, the choice of the EMA is to maintain an important academic link by integrating schooling into the care and considering it as a care. This school care is then progressive, focused on the fundamental subjects to begin with and above all without any performance objectives. The challenges are then to regain one's place as a teenager, to project oneself into the future, and to regain confidence and self-esteem. The school is part of the treatment plan and is sometimes the first point of interest for many adolescents who are in denial of their problems out of fear of needing psychiatric help.

Unlike other child psychiatry departments, the Salvator Pedagogical Space, managed by a National Education Coordinator, is not only a support for the pursuit of schooling but can be an actual schooling environment, allowing for the enrollment at NHSC (National Home-Schooling Center), for normal or adapted programs lasting for 1 or 2 years. A team of permanent teachers, as well as a team of external teachers, working within the framework of the Home Pedagogical Assistance Department (SAPAD) and a primary level teacher responsible for methodological 
assistance and the NHSC, create the structure which is the center of examination for the Brevet des collèges and Bac (middle school and high school diplomas). A nurse, a school doctor, and a teacher with experience in both health and education facilitate a natural link between care and schooling within the EMA.

The Pedological Space manages an active line of 369 adolescents, including 209 new patients. Among these, $53.11 \%$ have SRB, with a higher proportion of younger adolescents, with $48 \%-61 \%$ of them being middle school students. Pedagogical support is done in individual or small groups and the National Education team participates with the day hospital staff so that they can be accompanied back to school in conjunction with nursing or educational staff.

Returning to school is the goal of directly visible care and it is often the only initial request from families and sometimes the adolescent when he or she is better. It is gradually being done, through the NHSC, and is spread over 1-2 years or by requesting a program adapted with SAPAD hours to reconnect with their teachers. It may take 1-2 years for the patient to become an ordinary adolescent again, i.e., a student.

Returning to an institution is often much more complicated and must be preceded by the treatment of the delayed sleep phase syndrome that can be done in a day hospital or in a short hospital stay of a week to reset biological rhythms. It must not be done too soon and must be carefully organized. The choice of the school and the temporary status must be considered: rather at the beginning of the year and ideally at an orientation level (entry into high school in second or first year when choosing a course, in faculty). Timetable arrangements can be made especially if the break has been brief and if the affected adolescent returns to their establishment full time. It seems important to us that the student should be received by the school principal and the senior educational adviser, accompanied by a caregiver who knows both the places and the people who are listening to them. This can help decrease their anxiety.

It should be emphasized, however, that our experience of integrated management within the EMA shows that the secondary benefits of SRB for adolescents are to stay at home with attentive parents but also for some the operation is entirely organized around the SRB and can be a barrier against returning to an ordinary school environment. Therefore, the type of care we propose seems to us to be necessary in severe cases of SRB, but it probably does not suit all situations, at the risk that its sometimes too comfortable appearance may ultimately hinder healing. We also stress that it is essential to minimize this aspect of comfort, to start exposures as soon as the adolescent's condition allows it again.

In conclusion, we can say that the innovative integration of a school within an acute care structure allows the EMA to positively use the school in all its fundamental dimensions to promote the healthy development 
of an adolescent, as a fully-fledged care tool in relation to the more traditional methods of care used in child psychiatry. The sustainability and the extension of this system, which is now fully integrated into our care methods, seems to us to be essential to the successful completion of our child psychiatry missions.

Conflict of interest: The authors declare no conflicts of interest.

\section{REFERENCES}

1. Aloi M, Segura-García C. Anxiety and depression mediate the role of low self-esteem and low self-directedness in the development of eating disorders. Int J Adolesc Med Health 2016 Oct 14.

2. Bat-Pitault F, Da Fonseca D. What are the effects and management of short sleep duration in adolescents? Arch Pediatr 2012;19(10):1095-1099.

3. Byrne M, Eichen DM, Fitzsimmons-Craft EE, Taylor CB, Wilfley DE. Perfectionism, emotion dysregulation, and affective disturbance in relation to clinical impairment in college-age women at high risk for or with eating disorders. Eat Behav 2016;23:131-136.

4. Cain N, Gradisar M. Electronic media use and sleep in school-aged children and adolescents: A review. Sleep Med 2010;11(8):735-742.

5. Carskadon MA. Sleep's effects on cognition and learning in adolescence. Prog Brain Res 2011;190:137-143.

6. Carskadon MA, Vieira C, Acebo C. Association between puberty and delayed phase preference. Sleep 1993;16(3):258-262.

7. Challamel M, Franco P, Hardy M. Troubles du sommeil de l'adolescent. Le sommeil de I'enfant. Paris: Elsevier, 2009:51-57.

8. Chambry J, Agman G. L'anorexie mentale masculine à I'adolescence. Psychiatr Enfant 2006;49(2):47-511.

9. Dahl R, Lewin D. Pathways to adolescent health: sleep regulation and behavior. J Adolesc Health 2002;31:175- 184.

10. DeJong $H$, et al. Quality of life in anorexia nervosa, bulimia nervosa and eating disorder not-otherwise-specified. J Eat Disord 2013;1:43.

11. Fairburn CG, Harrison PJ. Eating disorders. Lancet 2003;361(9355):407-16.

12. Flament $M$, Buchholz A, Henderson $K$, et al. Comparative distribution and validity of DSM-IV and DSM-5 diagnoses of eating disorders in adolescents from the community. Eur Eat Disord Rev. 2015;23(2):100-10.

13. Gallé-Tessonneau M, Doron J, Grondin O. Des critères de repérage aux statégies thérapeutiques de prise en charge du refus scolaire: une revue de la littérature internationale systématique. Pratiques psychologiques. 2017;23:1-19.

14. Godart N, et al. Epidemiology of anorexia nervosa in a French community-based sample of 39,542 adolescents. Open Journal of Epidemiology 2013;3:53-61. 
15. Gradisar M, Gardner G, Dohnt H. Recent worldwide sleep patterns and problems during adolescence: a review and meta-analysis of age, region and sleep. Sleep Med 2011;12(2):110-118.

16. Guénolé $F$, et al. Sommeil et substances à l'adolescence: les effets de la caféine, de l'alcool, du tabac et du cannabis. Med Sommeil 2011;8:152-158.

17. Hansen M, Janssen F, Schiff A, Zee PC, Dubocovich ML. The impact of school daily schedule on adolescent sleep. Pediatrics 2005;115(6):1555-1561.

18. HAS. Recommandations - Anorexie mentale: prise en charge. wwwhas-santefr/portail/ jcms/c_985715/fr/anorexie-mentale-prise-en-charge30sept2010.

19. Hoek HW. Incidence, prevalence and mortality of anorexia nervosa and other eating disorders. Curr Opin Psychiatry 2006;19(4):389-394.

20. Ingul J, Nordahl H. Anxiety as a risk factor for school absenteism: what differentiates anxious school attenders from non-attenders? Annals of General Psychiatry 2013;12(1):25.

21. Jeammet P. Anorexie boulimie : les paradoxes de l'adolescence. Paris : Hachette, 2011.

22. Kopasz M, et al. Sleep and memory in healthy children and adolescents - A critical review. Sleep Med Rev 2010;14(3):167-177.

23. Lock J, Reisel B, Steiner H. Associated health risks of adolescents with disordered eating: how different are they from their peers? Results from a high school survey. Child Psychiatry Hum Dev 2001;31(3):249-265.

24. Millman R. Excessive sleepiness in adolescents and young adults: causes, consequences and treatment strategies. Pediatrics 2005;115(6):1774-1786.

25. Muise AM, Stein DG, Arbess G. Eating disorders in adolescent boys: a review of the adolescent and young adult literature. J Adolesc Health 2003;33(6):427-435.

26. Munezawa T, et al. The association between use of mobile phones after lights out and sleep disturbances among Japanese adolescents: a nationwide cross-sectional survey. Sleep 2011;34(8):1013-1020.

27. Ohayon M. Prévalence et comorbidité des troubles du sommeil dans la population générale. Rev Prat 2007;57:1521-1528.

28. Richards $\mathrm{H}$, Hadwin J. An exploration of the relationship between trait anxiety and school attendance in young people. School Mental Health 2011;3(4):236- 244.

29. Roberts $R$, Roberts $C$, Duong H. Sleepless in adolescence: prospective data on sleep deprivation, health and functioning. J Adolesc Health 2009;32(5):1045-57.

30. Sundquist J, et al. School Achievement and Risk of Eating Disorders in a Swedish National Cohort. Journal of the American Academy of Child \& Adolescent Psychiatry. 2016;55(1): 41-6.e1.

31. Suzuki $\mathrm{H}$, et al. Clarification of the factor structure of the 12-item General Health Questionnaire among Japanese adolescents and associated sleep status. Psychiatry Res 2011;188(1):138-146.

32. Tchanturia K, Campbell IC, Morris R, Treasure J. Neuropsychological studies in anorexia nervosa. Int J Eat Disord 2005;37 Suppl:S72-6.

33. Vannucci $A$, et al. An examination of the clinical impairment assessment among women at high risk for eating disorder onset. Behaviour Research and Therapy 2012;50(6):407-414.

34. Wolfson A, Carskadon M. Understanding adolescents sleep patterns and school performance: a critical appraisal. Sleep Med Rev 2003;7(6):491-506. 